\title{
XXIX. Researches in the undulatory theory of light, continued; on the Elliptical polarization produced by quartz. Part I
}

\section{J. Tovey Esq.}

To cite this article: J. Tovey Esq. (1839) XXIX. Researches in the undulatory theory of light, continued; on the Elliptical polarization produced by quartz. Part I, Philosophical Magazine Series 3, 14:88, 169-174, DOI: 10.1080/14786443908649701

To link to this article: http://dx.doi.org/10.1080/14786443908649701

曲 Published online: 01 Jun 2009.

Submit your article to this journal $[\pi$

Џll Article views: 2

Q View related articles $\sqsubset$ 
XXIX. Researches in the Undulatory Theory of Light, continued; on the Elliptical Polarization produced by Quartz. Part I. By J. Tovex, Esq.

To the Editors of the Philosophical Magazine and Journal. Gentlemen,

A $\mathrm{T}$ the conclusion of my paper on the Cause of the EllipA tical Polarization of Light, (L. and E. Phil. Mag., vol. xii. p. 10,) I stated that $I$ intended to apply my formulæ to the case of this phænomenon produced by quartz crystal. The fulfilment of this intention has been long delayed; but I now proceed to the subject.

In the first place it is needful to put the last four of the expressions (3.), of the paper referred to, into a more convenient form, thus : by the rules of trigonometry,

and

$$
1-\cos k \Delta x=\operatorname{vers} k \Delta x,
$$

$\cos (k \Delta x-b)-\cos b=-\cos b$ vers $k \Delta^{\circ} x+\sin b \sin k \Delta x$, $\cos (k \Delta x+b)-\cos b=-\cos b$ vers $k \Delta x-\sin b \sin k \Delta x$, $\sin (k \Delta x-b)+\sin b=+\sin b$ vers $k \Delta x+\cos b \sin k \Delta x$, $\sin (k \Delta x+b)-\sin b=-\sin b \operatorname{vers} k \Delta x+\cos b \sin k \Delta x ;$ hence if we put

$$
\begin{aligned}
& m \Sigma \cdot \psi(r) \Delta y \Delta z \text { vers } k \Delta x=\sigma, \\
& m \Sigma \cdot \psi(r) \Delta y \Delta z \text { sin } k \Delta x=\sigma^{\prime},
\end{aligned}
$$

the expressions referred to will become

$$
\begin{gathered}
-\sigma \cos b+\sigma^{\prime} \sin b=s_{2}, \\
-\sigma \cos b-\sigma^{\prime} \sin b=s_{2}^{\prime}, \\
\sigma^{\prime} \cos b+\sigma \sin b=s_{3}, \\
\sigma^{\prime} \cos b-\sigma \sin b=s_{3}^{\prime} .
\end{gathered}
$$

Now the third and fourth of the equations (4.) * $^{*}$ give $s_{l} s_{1}^{\prime}=s_{3} s_{3}^{\prime}$; hence $s_{l} s_{l}^{\prime}=\sigma^{\prime 2} \cos ^{2} b^{\prime}-\sigma^{2} \sin ^{2} b$, and therefore

$$
\cos b= \pm \sqrt{\frac{s_{1} s^{\prime}+\sigma^{2}}{\sigma^{\prime 2}+\sigma^{2}}} .
$$

The third of the equations (4.), and the value of $s_{3}^{\prime}$ in (11.) give

$$
\rho=-\frac{s_{i}}{\sigma^{\prime} \cos b-\sigma \sin b} \text {. }
$$

Let $\cos b$ and $-\cos b$ denote the two values of $\cos b$ given by (12.); these values will afford, by (13.), two corresponding values of $\rho$, which denote by $\rho_{1}$ and $\varrho_{2}$. Now either pair of these values satisfies the conditions of the problem; hence paper.

* The equations numbered from (1.) to (9.) inclusive, are in the former 
the first and second of the equations (4.) give, by taking the values of $s_{2}, s_{2}^{\prime}$, from (11.)

$$
\begin{aligned}
& n^{2}+s-\rho_{1}\left(\sigma \cos b+\sigma^{\prime} \sin b\right)=0, \\
& n^{2}+s+\rho_{2}\left(\sigma \cos b-\sigma^{\prime} \sin b\right)=0, \\
& n^{2}+s^{\prime}-\frac{\sigma \cos b-\sigma^{\prime} \sin b}{\rho_{1}}=0, \\
& n^{2}+s^{\prime}+\frac{\sigma \cos b+\sigma^{\prime} \sin b}{\rho_{2}}=0:
\end{aligned}
$$

because the sign of the sine of an arc remains the same while that of the cosine changes.

For the sake of abridgement put

$$
\begin{aligned}
& \phi(r)+\psi(r) \Delta y^{q}=p, \\
& \phi(r)+\psi(r) \Delta z^{2}=p^{\prime}, \\
& \psi(r) \Delta y \Delta z=q, \\
& m \Sigma \cdot p \Delta x=\mathbf{A}, \cdot \quad m \Sigma \cdot p^{\prime} \Delta x=\mathbf{A}^{\prime} \text {, } \\
& \frac{m}{2} \Sigma \cdot p \Delta x^{2}=\mathrm{A}_{i}, \quad \frac{m}{2} \Sigma \cdot p^{\prime} \Delta x^{2}=\mathrm{A}_{l}^{\prime} \text {, } \\
& \frac{m}{2.3} \Sigma \cdot p \Delta x^{3}=A_{2}, \quad \frac{m}{2.3} \Sigma \cdot p^{\prime} \Delta x^{3}=\mathbf{A}_{2}{ }^{\prime} \text {, } \\
& \frac{m}{2.3 .4 .} \Sigma \cdot p \Delta x^{4}=A_{3}, \quad \frac{m}{2.3 .4} \Sigma \cdot p^{\prime} \Delta x^{4}=A_{3}{ }^{\prime} \text {, } \\
& \& c . \\
& \& c . \\
& m \Sigma \cdot q \Delta x=\mathrm{B} \text {, } \\
& \frac{m}{2} \Sigma \cdot q \Delta x^{2}=\mathrm{B}_{l} \text {, } \\
& \frac{n}{2.3} \Sigma \cdot q \Delta x^{3}=\mathrm{B}_{2} \text {, } \\
& \text { \&c. }
\end{aligned}
$$

Then, since

$$
\begin{aligned}
& \sin k \Delta x=k \Delta x-\frac{k^{3} \Delta x^{3}}{2.3}+\& \mathrm{c} ., \\
& \cos k \Delta x=1-\frac{k^{3} \Delta x^{2}}{2}+\frac{k^{4} \Delta x^{4}}{2.3 .4}-\& \mathrm{c} ., \\
& \text { vers } k \Delta x=1-\cos k \Delta x,
\end{aligned}
$$

the formulæ (3.) and (10.) give

$$
\begin{aligned}
& s=-\mathrm{A}_{1} k^{2}+\mathrm{A}_{3} k^{4}-\& \mathrm{c} . \\
& s^{\prime}=-\mathrm{A} ! k^{2}+\mathrm{A}_{3}^{\prime} k^{4}-\& \mathrm{c} . \\
& s_{1}=\mathrm{A} k-\mathrm{A}_{2} k^{3}+\& \mathrm{c} . \\
& s_{1}^{\prime}=\mathrm{A}^{\prime} k-\mathrm{A}_{2}^{\prime} k^{3}+\& \mathrm{c} . \\
& \sigma=\mathrm{B}_{1} k^{2}-\mathrm{B}_{3} k^{4}+\& \mathrm{c} . \\
& \sigma^{\prime}=\mathrm{B} k-\mathrm{B}_{2} k^{3}+\& \mathrm{c} .
\end{aligned}
$$


Before we proceed further we must make a few observations on the nature and relative magnitudes of the quantities contained in the formulæ (17.) The capital letters denote quantities depending entirely on the nature of the medium; and by examining (15.) and (16.) we may see that $A_{\text {, }}$ and $\mathbf{A}_{3}$ are sums of which the terms are all positive; but we infer that the latter is almost indefinitely smaller than the former, because it involves a power of $\Delta x$ higher by two units, and $\Delta x$ can never exceed the radius of the sphere of influence of a molecule of the medium. The same observations apply to $\mathrm{A}_{1}^{\prime}$ and $\mathrm{A}_{3}^{\prime}$. The quantities $\mathrm{A}, \mathrm{A}_{2}, \ldots \mathrm{A}^{\prime}, \mathrm{A}_{2}^{\prime}, \ldots \mathrm{B}, \mathrm{B}, \mathrm{B}_{2}$, ... are sums in each of which the terms must be about half of them positive and half negative, hence these quantities must be comparatively small, and their real magnitudes can be learnt only by comparing with experiments the results of the calculation, the basis of which must originally be hypothetical.

The sum $B$, which is the principal quantity in the value of $\sigma$, is the sum which we have shown in art. 15, p. 426, vol. ix. can always be made to vanish, by taking the axes of $y$ and $z$ in proper directions. Let us suppose this done, and that the other sums in $\sigma$ are indefinitely small in comparison with $A_{1}, A_{;}^{\prime}$. Let us also suppose $\frac{B}{k}$ to be insensible. Then, by substituting the values (17.) in the equation (14.), writing $v$ for $\frac{n}{k}$, transposing, and stopping at the terms explicitly expressed in (17.), we get

$$
\begin{aligned}
v_{1}^{2} & =\mathbf{A}_{1}-\mathbf{A}_{3} k_{1}^{2}-\rho_{1} \sin b \cdot \mathbf{B}_{2} k_{l}, \\
& =\mathbf{A}_{1}^{\prime}-\mathbf{A}_{3} k_{1}^{2}+\frac{\sin b \cdot \mathbf{B}_{2} k_{1}}{\rho_{1}} \\
v_{2}{ }^{3}= & \mathbf{A}_{1}-\mathbf{A}_{3} k_{2}{ }^{2}-\rho_{2} \sin b \cdot \mathbf{B}_{2} k_{2} \\
& =\mathbf{A}_{1}^{\prime}-\mathbf{A}_{3}^{\prime} k_{2}{ }^{2}+\frac{\sin b \cdot \mathbf{B}_{2} k_{2}}{\rho_{2}}:
\end{aligned}
$$

where we have marked $v$ and $k$ with subscript figures, because the values of these quantities must necessarily be different in the two equations, while that of $n$ remains the same.

Suppose $\sigma^{2}$, and $\sigma \sin b$, to be indefinitely small in comparison with the quantities to which they are joined in (12.) and (13.), then

$$
\cos b= \pm \sqrt{\frac{s_{1} s_{1}^{\prime}}{\sigma^{\prime 2}}}
$$

and

$$
p=-\frac{s_{1}}{\sigma^{1} \cos b}
$$


hence, if we suppose $s_{1}=s_{1}^{\prime}$, the two values of $\rho$ will be $\rho_{1}$

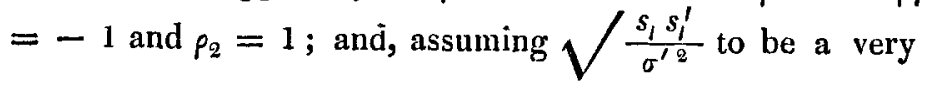
small quantity, we shall have $\sin b=1$ and $b=\frac{\pi}{2}$, nearly. Now, since $k=\frac{2 \pi}{\lambda}$, where $\lambda$ is the length of a wave, if these values be substituted in (18.), and the terms involving $A_{3}, A_{3}^{\prime}$, be comparatively insensible, there will result

$$
\begin{aligned}
& v_{1}^{2}=\mathrm{A}_{1}+\mathrm{B}_{2} \frac{2 \pi}{\lambda_{1}}, \\
& v_{2}^{2}=\mathrm{A}_{1}-\mathrm{B}_{2} \frac{2^{2} \pi}{\lambda_{2}} .
\end{aligned}
$$

Since $\frac{n}{k}=v$, and $k=\frac{2 \pi}{\lambda}$, the expressions (2.) may be changed to

$$
\begin{aligned}
& \eta=a \sin \left\{\frac{2 \pi}{\lambda}(v t-x)\right\}, \\
& \zeta=\rho a \sin \left\{\frac{2 \pi}{\lambda}(v t-x)-b\right\} .
\end{aligned}
$$

Now either of the values of $v$, and the corresponding value of $\rho$, may be substituted for $v$ and $\rho$ in these expressions. But since the equations (1.) are of the first degree, they may be satisfied not only by the values of $\eta$ and $\zeta$ corresponding to each value of $v$, but by taking for $\eta$ and $\zeta$ the sums of these particular values, in which we may change the value of $a$ as $v$ changes. Hence the equations (1.) may be satisfied by

$$
\begin{aligned}
& \eta=a_{1} \sin \left\{\frac{2 \pi}{\lambda_{1}}\left(v_{1} t-x\right)\right\}+a_{2} \sin \left\{\frac{2 \pi}{\lambda_{2}}\left(v_{2} t-x\right)\right\} \\
& \zeta=p_{1} a_{1} \sin \left\{\frac{2 \pi}{\lambda_{1}}\left(v_{1} t-x\right)-b\right\}+\rho_{2} a_{2} \sin \left\{\frac{2 \pi}{\lambda_{2}}\left(v_{2} t-x\right)-b\right\} .
\end{aligned}
$$

If in these expressions we give to $p_{1}, p_{2}$, and $b$, the values just assigned to them, namely, $-1,1$, and $\frac{\pi}{2}$, we shall have $\eta=a_{1} \sin \left\{\frac{2 \pi}{\lambda_{1}}\left(v_{1} t-x\right)\right\}+a_{2} \sin \left\{\frac{2 \pi}{\lambda_{2}}\left(v_{2} t-x\right)\right\}$, $\zeta=-a_{1} \cos \left\{\frac{2 \pi}{\lambda_{1}}(v, t-x)\right\}+a_{2} \cos \left\{\frac{2 \pi}{\lambda_{2}}\left(v_{2} t-x\right)\right\}$.

Now suppose a quartz crystal to have two parallel faces perpendicular to its axis. Take $x$ in the direction of the 
axis; and conceive a ray of light, polarized in a given plane, to fall on the crystal in the direction of $x$; causing a vibration of the molecules situated in the surface of the crystal, which may be represented by

$$
\eta=a \sin \cdot \frac{2 \pi}{\lambda} v t
$$

where $\lambda$ and $v$ are the length and velocity of the waves in the incident ray.

Suppose the origin of $x$ to be at the surface of the crystal on which the light falls : then, when $x$ is zero, the expressions (23.) must coincide with (24.), which they will do if we make,

$$
a_{1}=a_{2}=\frac{a}{2}, \frac{v_{1}}{\lambda_{1}}=\frac{v_{2}}{\lambda_{2}}=\frac{v}{\lambda} .
$$

Let $\varepsilon=\sqrt{ }\left(\eta^{2}+\zeta^{2}\right)$, then $\varepsilon$ is the actual displacement of a molecule within the crystal; and if we denote by $\alpha$ the angle which $\varepsilon$ makes with $\eta$, then

$$
\tan \alpha=\frac{\zeta}{\eta}
$$

therefore, since $a,=a_{2}$, the expressions (23.) give

and hence

$$
\begin{aligned}
\tan \alpha & =\frac{\cos \cdot \frac{2 \pi}{\lambda_{2}}\left(v_{2} t-x\right)-\cos \cdot \frac{2 \pi}{\lambda_{1}}\left(v_{1} t-x\right)}{\sin \cdot \frac{2 \pi}{\lambda_{2}}\left(v_{2} t-x\right)+\sin \cdot \frac{2 \pi}{\lambda_{1}}\left(v_{1} t-x\right)} \\
& =\tan \cdot 2 \pi x\left(\frac{1}{\lambda_{1}}-\frac{1}{\lambda_{2}}\right) ; \\
\text { hence } \quad & \alpha=2 \pi x\left(\frac{1}{\lambda_{1}}-\frac{1}{\lambda_{2}}\right) .
\end{aligned}
$$

Now the equations (21.) give us

$$
\begin{aligned}
& v_{1}=\mathrm{A}_{1}^{\frac{1}{2}}+\frac{\mathrm{B}_{2}}{\mathrm{~A}^{\frac{1}{2}}} \frac{\pi}{\lambda_{1}}, \\
& v_{2}=\mathrm{A}_{1}^{\frac{1}{t}}-\frac{\mathrm{B}_{2}}{\mathrm{~A}_{1}^{\frac{1}{3}}} \frac{\pi}{\lambda_{2}} ;
\end{aligned}
$$

nearly: therefore, since $\frac{v_{1}}{\lambda_{1}}=\frac{v_{2}}{\lambda_{2}}$, we find

$$
\frac{\mathrm{A}_{1}^{\frac{1}{2}}}{\lambda_{1}}+\frac{\mathrm{B}_{2}}{\mathrm{~A}_{1}^{\frac{1}{2}}} \frac{\pi}{\lambda_{1}^{z_{1}}}=\frac{\mathrm{A}_{1}^{\frac{1}{2}}}{\lambda_{\mathrm{g}}}-\frac{\mathrm{B}_{2}}{\mathrm{~A}_{\frac{2}{2}}^{\frac{z_{1}}{2}}} \frac{\pi}{\lambda_{2}^{2}} ;
$$

hence $\frac{1}{\lambda_{1}}-\frac{1}{\lambda_{2}}=-\pi \frac{B_{2}}{A^{\frac{1}{2}}}\left(\frac{1}{\lambda_{1}^{2}}+\frac{1}{\lambda_{2}^{z}}\right)$

$$
=-2 \pi \frac{B_{2}}{A_{i}^{\frac{1}{3}}} \frac{1}{\lambda^{2}} \text {, nearly; }
$$


which gives

$$
\alpha=-4 \pi^{2} \cdot \frac{\mathrm{B}_{2}}{\mathrm{~A}_{i}^{i}} \frac{x}{\lambda^{2}} .
$$

From this result we learn, 1 st, that since $\alpha$ is the same for all values of $t$, every molecule vibrates to and fro in a straight line; 2nd, that $\alpha$ varies as $\frac{x}{\lambda^{2}}$, that is, directly as the thickness of the crystal through which the ray has passed, and inversely as the square of the length of the wave.

These results are equivalent to the well-known experimental laws of Biot; hence we conclude that our formulæ (23.), (25.), (26.), (27.), represent correctly the motions which constitute a ray of light passing through the crystal in the direction of its axis.

On looking at the length of this paper I think it will be best to reserve the remainder of the investigation for another.

I am, Gentlemen, yours, \&c.

Littlemoor, Clitheroe, Jan. 9, 1839.

JoHN Tovey.

P.S. In the paper, vol. xii. p. 11, line 8, for $\phi(r)$ read $\phi(r) \Delta \eta$; ibid. line 9 , for $\phi(r)$ read $\phi(r) \Delta \zeta$; and p. 12, equations (7.) for $\rho_{11} a_{11}\left(n_{11} t-k x-b\right)$, read $\rho_{11} a_{11} \sin \left(n_{11} t\right.$ $-k x-b)$. The corrections of a few other errors in the same paper have been previously given.

XXX. On a remarkable Property of Arteries considered as a Cause of Animal Heat. By J. M. WIn , M.D.*

A BOUT three years since whilst making a few experiments $A$ with caoutchouc, I was forcibly struck with the property it possesses of evolving heat when suddenly stretched, and was led at the time to infer the probability of other bodies being similarly endowed. The elastic coat of arteries especially, from the mechanical resemblance it bears to caoutchouc, appeared to be one of the substances most likely to exhibit this calefactory principle; and in the event of this being the case it would not be unreasonable to conclude that the incessant contractions and dilatations of the arteries during life must prove an efficient source of animal heat.

During the past week I was induced to resume the subject afresh, and upon' making an experiment with part of the aorta of a bullock, I felt much gratification in being able to verify my previous conjecture. The experiment was per-

* Communicated by the Author. 